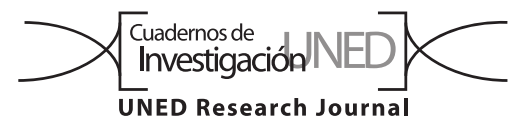

\title{
¿Serán vulnerables los bosques tropicales secos a los cambios climáticos, y cuáles serán sus efectos sociales?
}

\author{
Jennifer S. Powers
}

University of Minnesota, Departments of Ecology, Evolution and Behavior and Plant and Microbial Biology, 1479 Gortner Lab, 140 Gortner

Ave, Saint Paul, MN 55108 USA; powers@umn.edu

Recibido 28-VI-2018 • Corregido 15-IX-2018 • Aceptado 14-I-2019

\begin{abstract}
Will tropical dry forests be vulnerable to climate change, and what will be their social effects? Tropical dry forests provide societies with many valuable ecosystem services. These forests are characterized by alternating rainy and dry seasons. Changes to rainfall regimes are likely to negatively impact the internal dynamics of dry forests; at longer timescales, climate change may shift the geographical borders of dry forests. Society should establish policies and management practices such as reforestation with drought tolerant tree species to adapt to these changes now.
\end{abstract}

Key words: climate change, dry tropical forest, ecosystem services, adaptation.
RESUMEN: Los bosques tropicales secos proporcionan a las sociedades muchos servicios ecosistémicos valiosos. Estos bosques se caracterizan por alternar estaciones lluviosas y secas. Es probable que los cambios en los regímenes de lluvia tengan un impacto negativo en la dinámica interna de los bosques secos. A mayor plazo, el cambio climático puede cambiar las fronteras geográficas de estos bosques. La sociedad debería establecer desde ahora políticas y prácticas de gestión -como la reforestación con árboles tolerantes a la sequía- para adaptarse a estos cambios.

Palabras clave: cambio climático, bosque tropical seco, servicios ecosistémicos, adaptación.
¿Qué son los bosques tropicales secos? Son bosques que se distinguen de los bosques tropicales húmedos por una menor precipitación; sin embargo, la lluvia que reciben cae durante distintas estaciones húmedas que se alternan con las estaciones secas (Murphy \& Lugo, 1986). Esta estacionalidad trae como consecuencia una abundancia alta de especies caducifolias (que pierden sus hojas durante la estación seca) (Fig. 1). Los bosques secos son extensos a través de los trópicos (Miles et al., 2006), y muchas personas dependen de ellos y de los servicios ecosistémicos que brindan para su sustento (Maass et al., 2005). En este artículo, reviso la evidencia de cómo los bosques secos pueden responder al cambio climático actual y futuro, los factores regionales o específicos del sitio que modifican estos procesos y las posibles consecuencias para la sociedad (Fig. 2).

\section{CAMBIO CLIMÁTICO EN LOS TRÓPICOS}

Hay abundante evidencia de que el clima está cambiando debido a las emisiones humanas de gases con efecto invernadero a la atmósfera (Cubasch et al., 2013). En los trópicos, los climas futuros serán más cálidos con regímenes de lluvia menos predecibles (Dai, 2013), temporadas secas extendidas (p.e., en la Amazonia; Fu et al., 2013), y menos Iluvia en Centroamérica y México (Karmalkar, Bradley \& Diaz, 2011). La mayor parte de la investigación respecto al cambio climático y los bosques tropicales se ha centrado en los bosques tropicales húmedos (Corlett, 2011; Wood, Cavaleri \& Reed, 2012; Olivares, Svenning, van Bodegom \& Balslev, 2015; Corlett, 2016; Cusack et al., 2016). Sin embargo, la literatura respecto a cómo responderán los bosques tropicales secos al cambio climático es aún escasa, aunque está aumentando (Allen et al., 2017). 

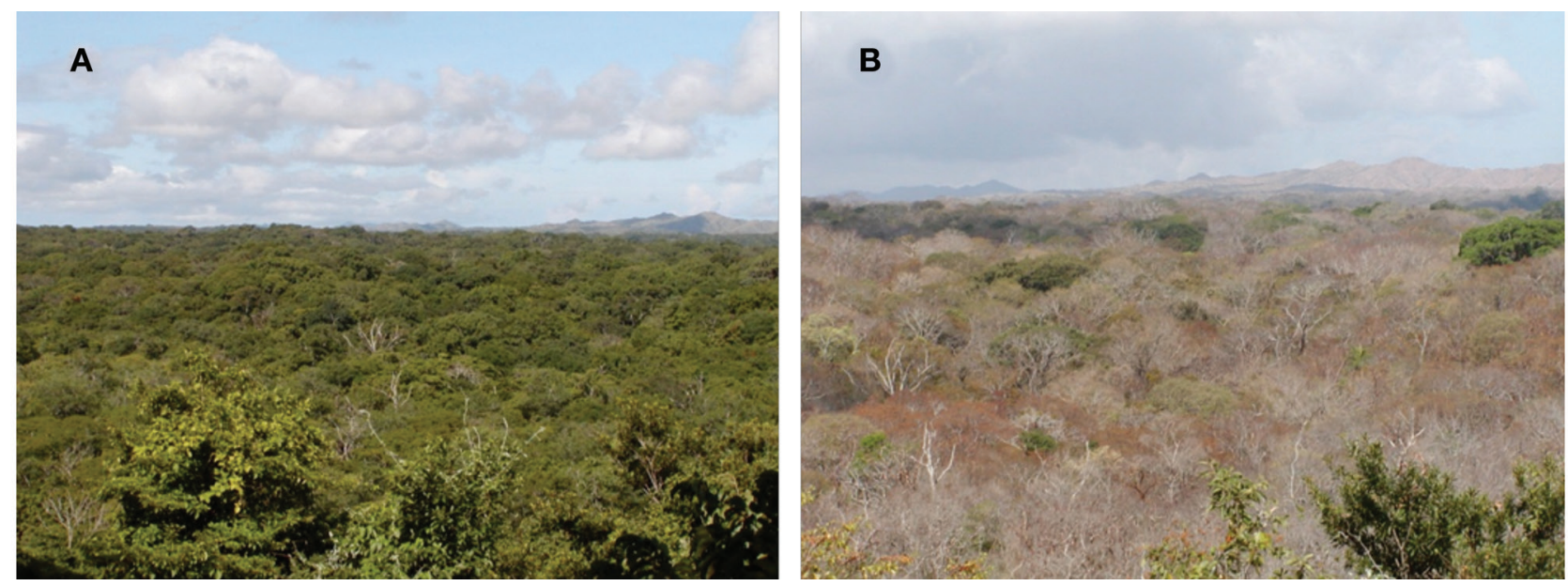

Fig. 1. Vista del mirador del Área de Conservación Guanacaste, Sector Santa Rosa en la estación lluviosa (a) y la seca (b) (fotos de Jennifer Powers).

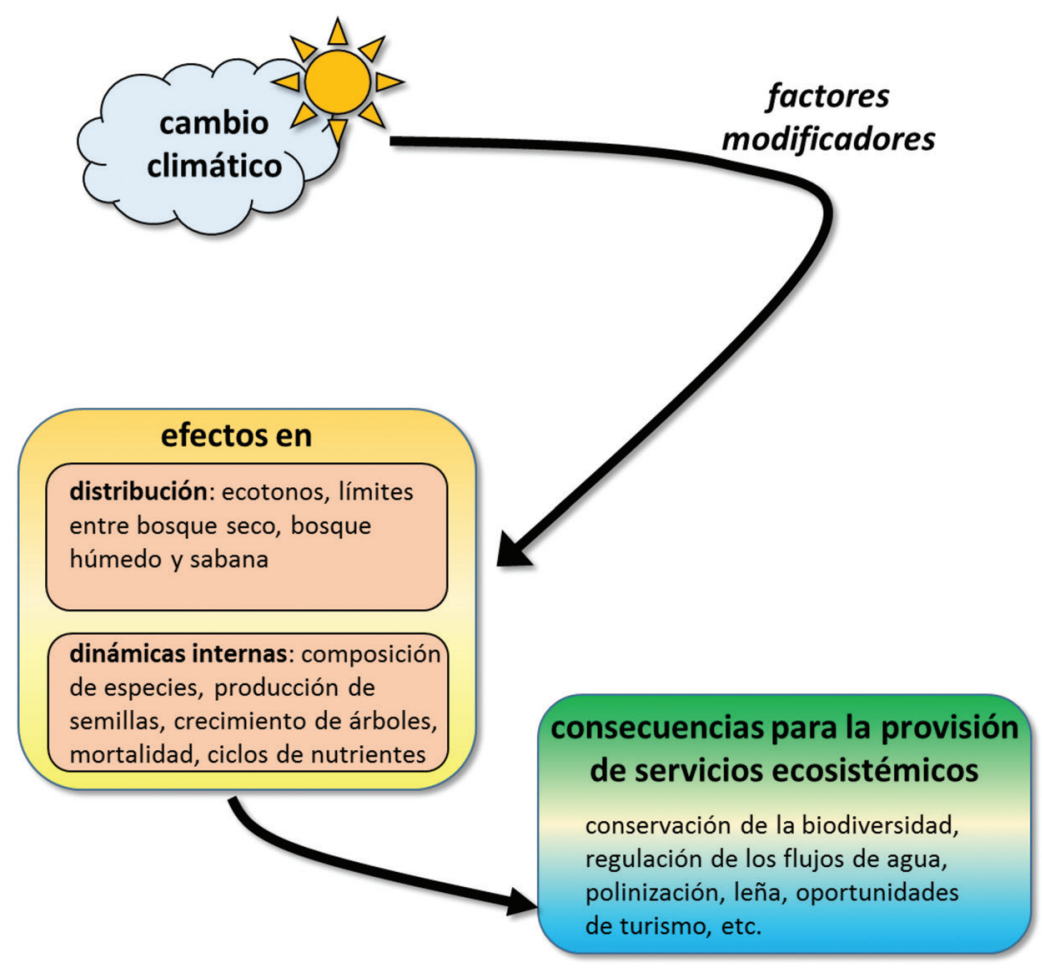

Fig. 2. Modelo conceptual de cómo el cambio climático afecta los tropicales bosques secos, los servicios ecosistémicos que brindan y los factores que modifican los efectos del cambio climático.

\section{BOSQUES SECOS Y CAMBIO CLIMÁTICO}

El cambio climático puede afectar los procesos de las comunidades ecológicas y los del ecosistema dentro de un bosque seco dado y, también, en escalas de tiempo más largas afectar la ubicación geográfica futura del bosque seco tropical en relación con otros biomas como sabanas o bosques húmedos (Enquist, 2002; Malhi et al.,
2009). A continuación, analizo la investigación en estas dos escalas espaciales distintas.

\section{A. Los efectos del cambio climático sobre de los bos- ques tropicales secos}

No se sabe con seguridad cómo los bosques tropicales secos responderán a climas cambiantes. Sin embargo, es probable que sus respuestas sean diferentes a la de 
los bosques tropicales húmedos debido a la diferente fenología de las copas de los árboles (Eamus, 1999) y los diferentes procesos hidrológicos dominantes que afectan el flujo de agua (Farrick \& Branfireun, 2013). Por ejemplo, un estudio utilizó un modelo dinámico de vegetación para evaluar cómo los aumentos de temperatura y la disminución de la lluvia afectan a los bosques húmedos y secos en Bolivia (Seiler, Hutjes, Kruijt \& Hickler, 2015). Concluyeron que las reservas de carbono en la vegetación eran más sensibles a los cambios en el clima en los bosques secos que en los húmedos; además, los cambios en los bosques húmedos se debieron a una combinación de temperatura y precipitación, mientras que en los bosques secos los cambios fueron en respuesta a los regímenes de precipitación (Seiler et al., 2015). Además, las presiones humanas como la deforestación, la fragmentación y la presión demográfica afectan aún más la vulnerabilidad de los bosques tropicales al cambio climático, y estos factores difieren entre los bosques secos y húmedos (Eguiguren-Velepucha et al., 2016). En conjunto, estos estudios sugieren que no podemos inferir la vulnerabilidad del bosque seco al cambio climático a partir de estudios realizados en su contraparte húmeda.

Una síntesis de literatura reciente evaluó la evidencia de si el bosque tropical seco será vulnerable o resistente a los cambios esperados en la precipitación (Allen et al., 2017). Los autores consideraron dos hipótesis alternativas: 1) los bosques tropicales secos serán resistentes o resilientes a la disminución de las precipitaciones porque las especies ya están adaptadas a las sequías estacionales prolongadas, y 2) los bosques secos serán vulnerables a las mayores sequías porque ya están cerca de los límites hidrológicos. Este trabajo mostró que la dinámica forestal interna, como el crecimiento arbóreo (Mendivelso, Camarero, Royo Obregón, Gutiérrez \& Toledo, 2013), el reclutamiento de plántulas (Maza-Villalobos, Poorter \& MartinezRamos, 2013) y la mortalidad arbórea (Imbert \& Portecop, 2008) se vieron afectados por la variación en la lluvia total anual. Por lo tanto, Allen y colaboradores concluyeron que los bosques tropicales secos serán sensibles a los cambios futuros en la cantidad y / o el momento de la lluvia. Además, dado que las especies varían en sus respuestas a la lluvia (Mendivelso et al., 2013), podemos esperar que la composición de especies de los bosques tropicales secos cambie en respuesta al cambio actual así como el esperado en el clima, y que las especies más vulnerables disminuyan su abundancia.

Sin embargo, es importante darse cuenta de que no todos los bosques tropicales secos experimentarán el mismo cambio climático, no todos los bosques secos responderán de la misma manera, y algunos efectos del cambio climático no son directos (Fig. 2). Estudios recientes han enfatizado la variación en la composición de especies arbóreas, la biogeografía, los suelos, la topografía y la estacionalidad de las lluvias en los bosques tropicales secos (Powers, Becknell, Irving \& Pèrez-Aviles, 2009; Balvanera, Quijas \& Perez-Jimenez, 2011; Banda-R. et al., 2016). Además de la gestión de los bosques (Eguiguren-Velepucha et al., 2016), es muy probable que estos factores modifiquen la forma en que este ecosistema responderá al cambio climático (Fig. 2). Por último, el cambio climático puede afectar los ecosistemas directamente, por ejemplo, a través del aumento de las tasas de mortalidad de árboles a causa de las sequías (Allen et al., 2017). El cambio climático también puede afectar indirectamente a los ecosistemas a través de cambios en los regímenes de perturbación. Las perturbaciones importantes en los bosques secos incluyen incendios, vendavales y huracanes. Los efectos de los cambios en los regímenes de perturbación en los servicios de los ecosistemas y la resiliencia de los bosques se han estudiado más en los bosques templados y boreales (Seidl, Spies, Peterson, Stephens \& Hicke, 2016) que en los secos.

\section{B. Controles sobre la ubicación de los bosques tropi- cales secos y las transiciones entre los biomas}

Además de los cambios en las condiciones internas y la dinámica de los bosques secos, es posible que el cambio climático altere la ubicación geográfica de los bosques tropicales secos en escalas de tiempo más largas. Tanto los modelos de distribución de especies como los modelos de nichos ecológicos (Enquist 2002; Prieto-Torres, Navarro-Sigüenza, Santiago-Alarcon \& Rojas-Soto, 2016, Aguirre et al., 2017) y modelos dinámicos de vegetación que simulan mecanismos de crecimiento arbóreo y dinámica forestal pueden proporcionar predicciones sobre si las áreas que actualmente ocupa la vegetación forestal serán adecuadas para este bioma en futuros escenarios climáticos.

Los análisis de los datos de la cubierta terrestre tropical en comparación con el déficit climatológico-- una medida de la aridez o la disponibilidad de agua-muestran que la distribución actual de los bosques secos es intermedia entre los más húmedos y las sabanas más secas (Malhi et al., 2009). Por lo tanto, es posible que los climas futuros con menos lluvia o una Iluvia más variable puedan dar como resultado la expansión de los bosques secos hacia áreas que actualmente ocupan bosques tropicales húmedos (Enquist, 
2002; Malhi et al., 2009). Otros estudios advierten que los límites entre los bosques tropicales y las sabanas son abruptos (Hirota, Holmgren, Van Nes \& Scheffer, 2011). Los bosques con distribuciones de lluvia actuales cerca de los puntos de inflexión entre estados estables alternativos (como sabanas o bosques secos) pueden ser particularmente vulnerables al cambio climático (Hirota et al., 2011). Por lo tanto, es posible que las áreas que actualmente contienen bosque tropical seco se conviertan en sabana si los climas se vuelven más secos. Es importante tomar en cuenta que factores como el cambio en el uso de la tierra, la fragmentación forestal, y la reducción de la abundancia y diversidad de polinizadores o dispersores de semillas pueden disminuir la capacidad de los biomas para moverse en respuesta al cambio climático (Aguirre et al., 2017).

\section{CONSECUENCIAS POTENCIALES PARA LA SOCIEDAD}

Los bosques tropicales secos proporcionan a las sociedades que viven dentro y alrededor de ellos muchos servicios ecosistémicos (Chopra, 1993; Maass et al., 2005; Kalaba, Quinn \& Dougill, 2013). Es probable que estos servicios vitales del ecosistema se vean afectados negativamente por el cambio climático. La siguiente es una lista parcial de algunos servicios ecosistémicos importantes proporcionados por el bosque seco tropical.

Acuíferos: el acuífero (depósito de agua subterránea) es la fuente dominante de agua para muchas personas en los ecosistemas de bosques secos, y aún existen muchas lagunas en nuestro conocimiento de cómo este recurso se verá afectado por el cambio climático (Farrick \& Branfireun, 2013).

Productos forestales no maderables: los bosques tropicales secos tienen una alta biodiversidad que los humanos usan directamente. Muchas personas extraen productos forestales no maderables, como frutas, hongos y miel de los bosques secos (Kalaba et al., 2013).

Leña y forraje: muchas personas dependen de los bosques tropicales secos para obtener leña para cocinar o forraje para los animales (Chopra, 1993). Si la sequía aumenta el estrés de las plantas, es probable que estos productos disminuyan.

Secuestro de carbono y conservación del suelo: los bosques tropicales secos almacenan grandes cantidades de carbono en el suelo y tienen el potencial de secuestrar aún más si todas las tierras que han sido deforestadas con el tiempo fueran restauradas (Becknell, Kissing \& Powers, 2012; Gamboa, Hidalgo, De Leon, Etchevers, Gallardo \& Campo, 2010). Además, algunos de los valores económicos más altos de los bosques secos se deben a sus funciones de conservación del suelo (Chopra, 1993). Si la biomasa disminuye con el aumento de la sequía, estos servicios del ecosistema se verán comprometidos.

\section{SOLUCIONES, ESTABLECIMIENTO DE UNA AGENDA PARA LA ADAPTACIÓN Y CONCLUSIONES}

El cambio climático está ocurriendo ahora; muchos bosques tropicales secos ya están mostrando signos de cambio. Las comunidades locales y las agencias gubernamentales que manejan los recursos de los bosques secos tienen ahora la oportunidad y el reto de garantizar ecosistemas resilientes de bosques secos para el futuro. Una dirección prometedora para la adaptación al cambio climático es identificar especies de árboles nativos que son resistentes a la sequía (Werden et al., 2017) o mezclas de especies que proveen grandes cantidades de servicios ecosistémicos (Hall, Ashton, Garen \& Jose, 2011). Estos deben ser utilizados en proyectos de restauración, así como en plantaciones forestales. Otro enfoque prometedor involucra la "restauración ajustada a la lluvia", es decir, las actividades de gestión del tiempo, tales como la siembra de semillas en momentos en que se pronostica que la precipitación es más alta (Sitters, Holmgren, Stoorvogel \& López, 2012). Por último, los gobiernos municipales y nacionales deben planificar para precipitaciones más variables y mayor frecuencia de sequías, porque esto tiene amplias implicaciones, más allá de los bosques secos, para actividades como la agricultura, los recursos hídricos para las personas, el turismo, etcétera. La comunidad internacional deberá proporcionar ayuda financiera y / o técnica para apoyar a muchos países que poseen bosques secos a adaptarse. En resumen, ahora es el mejor momento para actuar a fin de desarrollar adaptaciones robustas a los climas cambiantes.

\section{AGRADECIMIENTOS}

Agradezco a Andrea Vincent la oportunidad de presentar estas ideas en forma impresa y en una conferencia. Juan Manuel Dupuy Rada, Julio Campo, y un revisor anónimo tuvieron la amabilidad de proporcionar comentarios constructivos sobre el manuscrito. La financiación para este trabajo provino de US Department of Energy, Office of Science, Office of Biological and Environmental 
Research, Terrestrial Ecosystem Science (TES) Program award number DE-SC0014363.

\section{REFERENCIAS}

Aguirre, N., Eguiguren, P., Maita, J., Ojeda, T., Sanamiego, N., Furniss, M., \& Aguirre, Z. (2017). Potential impacts to dry forest species distribution under two climate change scenarios in southern Ecuador. Neotropical Biodiversity, 3,18-29.

Allen, K., Dupuy, J. M., Gei, M.G., Hulshof, C. M., Medvigy, D., Pizano, C., Salgado-Negret, B., Smith, C. M., Trierweiler, A., Van Bloem, S. J., Waring, B. G., Xu, X., Powers, J. S. (2017). Will seasonally dry tropical forests be sensitive or resistant to future changes in rainfall regimes? Environmental Research Letters, 12, 023001.

Balvanera, P., Quijas, S., \& Perez-Jimenez, A. (2011). Distribution Patterns of Tropical Dry Forest Trees Along a Mesoscale Water Availability Gradient. Biotropica, 43, 414-422.

Banda, K., Delgado-Salinas, A., Dexter, K. G., Linares-Palomino, R., Oliveira-Filho, A., Prado, D., ... \& Pennington, R. T. (2016). Plant diversity patterns in neotropical dry forests and their conservation implications. Science, 353,1383-1387.

Becknell, J. M., Kissing, L. B., \& Powers, J. S. (2012). Aboveground biomass in mature and secondary seasonally dry tropical forests: a literature review and global synthesis. Forest Ecology and Management, 276, 88-95.

Chopra, K. (1993). The value of non-timber forest products: an estimation for tropical deciduous forests in India. Economic Botany, 47, 251-257.

Corlett, R. T. (2011). Impacts of warming on tropical lowland rainforests. Trends in Ecology and Evolution, 26, 606-613.

Corlett, R. T. (2016). The impacts of droughts in tropical forests. Trends in Plant Science, 21, 584-593.

Cubasch, U., Wuebbles, D., Chen, D., Facchini, M. C., Frame, D., Mahowald, N., \& Winther, J.-G. (2013). Introduction. En: Stocker, T.F., D. Qin, G.-K. Plattner, M. Tignor, S.K. Allen, J. Boschung, A. Nauels, Y. Xia, V. Bex \& P.M. Midgley (Eds.), Climate Change 2013: The Physical Science Basis. Contribution of Working Group I to the Fifth Assessment Report of the Intergovernmental Panel on Climate Change. Cambridge, United Kingdom and New York, NY, USA: Cambridge University Press.

Cusack, D. F., Karpman, J., Ashdown, D., Cao, Q., Ciochina, M., Halterman, S., Lydon S., \& Neupane, A. (2016). Global change effects on humid tropical forests: Evidence for biogeochemical and biodiversity shifts at an ecosystem scale. Reviews of Geophysics, 54, 523-610.

Dai, A. G. (2013). Increasing drought under global warming in observations and models. Nature Climate Change, 3, 52-58.
Eamus, D. (1999). Ecophysiological traits of deciduous and evergreen woody species in the seasonally dry tropics. Trends in Ecology and Evolution, 14, 11-16.

Eguiguren-Velepucha, P. A., Maita Chamba, J. A., Aguirre Mendoza, N.A., Ojeda-Luna, T. L., Samaniego-Rojas, N.S., Furniss, M. J., Howe, C., \& Aguirre Mendoza, Z. H. (2016). Tropical ecosystems vulnerability to climate change in southern Ecuador. Tropical Conservation Science, 9, 1-17.

Enquist, C. A. F. (2002). Predicted regional impacts of climate change on the geographical distribution and diversity of tropical forests in Costa Rica. Journal of Biogeography, $29,519-534$.

Farrick, K. K. \& Branfireun, B. A. (2013). Left high and dry: a call to action for increased hydrological research in tropical dry forests. Hydrological Processes, 27, 3254-3262.

Fu, R., Yin, L., Li, W., Arias, P. A., Dickinson, R. E., Huang, L., ... \& Myneni, R. B. (2013). Increased dry-season length over southern Amazonia in recent decades and its implication for future climate projection. Proceedings of the National Academy of Sciences, 110, 18110-18115.

Gamboa, A. M., Hidalgo, C., De León, F., Etchevers, J. D., Gallardo, J. F., \& Campo, J. (2010). Nutrient addition differentially affects soil carbon sequestration in secondary tropical dry forests: Early- versus late-succession stages. Restoration Ecology, 18, 252-260.

Hall, J. S., Ashton, M. S., Garen, E. J., \& Jose, S. (2011). The ecology and ecosystem services of native trees: Implications for reforestation and land restoration in Mesoamerica. Forest Ecology and Management, 261, 1553-1557.

Hirota, M., Holmgren, M., Van Nes, E. H., \& Scheffer, M. (2011). Global resilience of tropical forest and savanna to critical transitions. Science, 334, 232-235.

Imbert, D., \& Portecop, J. (2008). Hurricane disturbance and forest resilience: Assessing structural vs. functional changes in a Caribbean dry forest. Forest Ecology and Management, 255, 3494-3501.

Kalaba, F. K., Quinn, C. H., \& Dougill, A. J. (2013). Contribution of forest provisioning ecosystem services to rural livelihoods in the Miombo woodlands of Zambia. Population and Environment, 35, 159-182.

Karmalkar, A. V., Bradley, R. S., \& Diaz, H. F. (2011). Climate change in Central America and Mexico: regional climate model validation and climate change projections. Climate Dynamics, 37, 605-629.

Maass, J. M., Balvanera, P., Castillo, A., Daily, G. C., Mooney, H. A., Ehrlich, P., ... \& Martínez-Yrizar, A. (2005).Ecosystem services of tropical dry forests: Insights from long-term ecological and social research on the Pacific Coast of Mexico. Ecology and Society, 10(1), art. 17

Malhi, Y., Aragão, L. E., Galbraith, D., Huntingford, C., Fisher, R., Zelazowski, P., ... \& Meir, P. (2009). Exploring the likelihood and mechanism of a climate-change-induced dieback of the Amazon rainforest. Proceedings of the National Academy of Sciences, 106(49), 20610-20615. 
Maza-Villalobos, S., Poorter, L., \& Martinez-Ramos, M. (2013). Effects of ENSO and temporal rainfall variation on the dynamics of successional communities in old-field succession of a tropical dry forest. PloS One, 8, http:// journals.plos.org/plosone/article?id=10.1371/journal. pone.0082040

Mendivelso, H. A., Camarero, J. J., Royo Obregón, O., Gutiérrez, E., \& Toledo, M. (2013). Differential growth responses to water balance of coexisting deciduous tree species are linked to wood density in a Bolivian tropical dry forest. PloS One, 8, e73855

Miles, L., Newton, A. C., DeFries, R. S., Ravilious, C., May, I., Blyth, S., ... \& Gordon, J. E. (2006).A global overview of the conservation status of tropical dry forests. Journal of Biogeography, 33, 491-505.

Murphy, P. G., \&. Lugo, A. E (1986). Ecology of tropical dry forest. Annual Review of Ecology and Systematics, 17, 67-88.

Olivares, I., Svenning, J.-C., van Bodegom, P. M., \& Balslev, H. (2015). Effects of warming and drought on the vegetation and plant diversity in the Amazon Basin. Botanical Review, 81, 42-69.

Powers, J. S., Becknell, J. M., Irving, J., \& Pèrez-Aviles, D. (2009). Diversity and structure of regenerating tropical dry forests in Costa Rica: geographic patterns and environmental drivers. Forest Ecology and Management, 258, 959-970.
Prieto-Torres, D. A., Navarro-Sigüenza, A. G., Santiago-Alarcon, D., \& Rojas-Soto, O. R. (2016). Response of the endangered tropical dry forests to climate change and the role of Mexican Protected Areas for their conservation. Global Change Biology, 22, 364-379.

Seidl, R., Spies, T. A., Peterson, D. L., Stephens, S. L., \& Hicke, J. A. (2016). REVIEW: Searching for resilience: addressing the impacts of changing disturbance regimes on forest ecosystem services. Journal of Applied Ecology, 53, $120-129$.

Seiler, C., Hutjes, R. W. A., Kruijt, B., \& Hickler, T. (2015). The sensitivity of wet and dry tropical forests to climate change in Bolivia. Journal of Geophysical Research-Biogeosciences, 120, 399-413.

Sitters, J., Holmgren, M., Stoorvogel, J. J., \& López, B. C. (2012). Rainfall-tuned management facilitates dry forest recovery. Restoration Ecology, 20, 33-42.

Werden, L. K., Alvarado, J., Zarges, S., Calderón, M., Schilling, E. M., Gutiérrez, L. M., \& Powers, J. S. (2017).Using soil amendments and plant functional traits to select native tropical dry forest species for the restoration of degraded Vertisols. Journal of Applied Ecology, 55(2), 10191028. DOI: 10.1111/1365-2664.12998

Wood, T. E., Cavaleri, M. A., \& Reed, S. C. (2012). Tropical forest carbon balance in a warmer world: a critical review spanning microbial- to ecosystem-scale processes. Biological Reviews, 87, 912-927. 